\title{
Effects of Bronchoalveolar Lavage on Refractory Mycoplasma pneumoniae Pneumonia
}

\author{
Yuanyuan Zhang MD, Ying Chen, Zhimin Chen MD PhD, Yunlian Zhou MD, \\ Yuanjian Sheng MD, Dan Xu PhD, and Yingshuo Wang MD
}

\begin{abstract}
INTRODUCTION: This study prospectively evaluated the effect of early bronchoalveolar lavage (BAL) on refractory Mycoplasma pneumoniae pneumonia with radiologically proven large pulmonary lesions in children. METHODS: A total of 35 children diagnosed as having refractory $M$. pneumoniae pneumonia with radiologically proven large pulmonary lesions completed the study. According to the time point of BAL, they were divided into 2 groups. In the early BAL group, BAL was performed within $3 \mathrm{~d}$ after admission $(n=22)$; in the late BAL group, BAL was performed $3 \mathrm{~d}$ later after admission $(n=13)$. Clinical effects were compared between these 2 groups. RESULTS: After BAL therapy, improvement in clinical symptoms and laboratory findings as well as resolution of pulmonary lesions on radiography were obtained in all subjects. The median fever duration after admission was $4(2-7) d$ and $5(2-10) d(P<.05)$, and the median hospitalization duration was 7 $(5-10) d$ and $10(5-14) d(P<.05)$, respectively, in the early BAL group and the late BAL group. Approximately $7 \mathrm{~d}$ after admission, $67 \%$ of subjects in the early BAL group showed resolution of atelectasis on $x$-ray image versus a corresponding rate of $14 \%$ in the late BAL group $(P<.05)$. Furthermore, laboratory indices recovered quicker in the early BAL group than in the late BAL group $(P<.01)$. CONCLUSIONS: BAL appeared to be efficacious and well-tolerated treatment for refractory $M$. pneumoniae pneumonia with radiologically proven large pulmonary lesions, and early application of BAL may be more beneficial. Key words: bronchoalveolar lavage; children; mycoplasma pneumonia; pneumonia; refractory; flexible bronchoscopy. [Respir Care 2014;59(9):1433-1439.

(C) 2014 Daedalus Enterprises]
\end{abstract}

\section{Introduction}

Mycoplasma pneumoniae is a common causative organism of respiratory infections in children., ${ }^{1,2}$ Although M. pneumoniae pneumonia is usually a benign self-limited disease, it may sometimes cause various extrapulmonary complications such as encephalitis, ${ }^{3}$ arthritis, ${ }^{4}$ pericarditis, ${ }^{5}$ and anemia, ${ }^{6}$ and can progress to a severe life-threat-

The authors are affiliated with the Department of Pulmonology, Children's Hospital, Zhejiang University School of Medicine, Hangzhou, People's Republic of China.

This research was partially supported by grants 81070004,81000765 , and 30872798 from the National Natural Science Foundation of China; grant LQ13H100002 from the Zhejiang Province Natural Science Foundation of China; grant 2013KYB152 from the Zhejiang Medicine and Health Research Fund; and grant Y201328580 from the Scientific Research Fund of Zhejiang Provincial Education Department. The authors have disclosed no conflicts of interest. ening pneumonia, such as necrotizing pneumonitis, ${ }^{7}$ ARDS, ${ }^{8}$ and fulminant pneumonia. ${ }^{9}$

For children, macrolides are the first choice agents for M. pneumoniae infections. However, there still are chances for aggravation of M. pneumoniae infection despite appropriate antibiotic therapy. These cases are usually defined as refractory $M$. pneumoniae pneumonia showing clinical and radiological deterioration despite macrolide antibiotic therapy for $7 \mathrm{~d}$ or longer. ${ }^{10,11}$ Although the underlying mechanisms are still uncertain, the macrolide-resistant M. pneumoniae infection and excessive immunological in-

\footnotetext{
Correspondence: Zhimin Chen MD PhD, Department of Pulmonology, Children's Hospital Zhejiang University School of Medicine, 57 Zhugan Xiang, Hangzhou 310003, People's Republic of China. E-mail: drchenzm@163.com.
}

DOI: $10.4187 /$ respcare. 03032 
flammation are most commonly proposed. ${ }^{12,13}$ Based on the latter mechanism, corticosteroids have been used with satisfactory therapeutic effect for children with refractory M. pneumoniae pneumonia. ${ }^{11,14,15}$ However, due to the potential risk of causing side effects and blurring diagnosis when using corticosteroids early in medication, it is advisable for physician to make decisions prudently.

Flexible fiberoptic bronchoscopy (FOB) was first reported to be performed in children $23 \mathrm{y}$ ago, ${ }^{16}$ and since then it has been widely accepted and applied. ${ }^{17-19}$ The main advantages of the FOB include 3 aspects. First, endobronchial abnormalities up to the fifth generation can be visualized directly. Second, samples from the lower airway can be obtained for bacteriologic, cytologic, and histologic detection with minimal damage. Third, FOB can offer additional therapeutic interventions, such as removing mucous plug or foreign body. ${ }^{20,21}$ Therefore, FOB with bronchoalveolar lavage (BAL) has now become an important means of diagnosis and therapy for respiratory diseases, including refractory pneumonia. However, until now there has been a paucity of data about BAL for the diagnosis and therapy of pediatric refractory $M$. pneumoniae pneumonia.

In this report, we prospectively studied 35 pediatric subjects with refractory $M$. pneumoniae pneumonia, who showed large lesions on their chest radiograph. Therapeutic BAL was adopted, and its clinical, laboratory, and radiological effects were analyzed.

\section{Methods}

\section{Subjects}

We conducted the study at Children's Hospital, Zhejiang University School of Medicine (Hangzhou, People's Republic of China). All the refractory M. pneumoniae pneumonia patients who were admitted to the hospital between January 1, 2013 and June 30, 2013 were eligible for the study. All the subjects had signs and symptoms indicative of pneumonia on admission, including fever, cough, abnormal lung auscultation, and a new infiltrate on chest radiograph. ${ }^{22}$ The diagnosis of $M$. pneumoniae infection was based on positive results for serologic test (M. pneumoniae immunoglobulin M-positive and antibody titer $\geq 1: 160$ ) and/or positive results for $M$. pneumoniae polymerase-chain-reaction tests of nasopharyngeal secretions or BAL. The diagnosis of refractory M. pneumoniae pneumonia was based on the presence of persistent fever and clinical as well as radiological deterioration after azithromycin treatment for $7 \mathrm{~d}$ or longer. ${ }^{10,11}$ In addition, to be eligible for recruitment, subjects were required to have large pulmonary lesions on radiological findings. Large lesion means that the extent of infiltration on chest radiography was more than one third of the lung. ${ }^{23}$ We ex-

\section{QUICK LOOK}

\section{Current knowledge}

Mycoplasma pneumonia is a common cause of respiratory infections in children. Refractory Mycoplasma pneumoniae pneumonia is defined as clinical and radiological deterioration despite macrolide antibiotic therapy for $7 \mathrm{~d}$. Refractory M. pneumoniae pneumonia is associated with systemic complications.

\section{What this paper contributes to our knowledge}

Therapeutic bronchoalveolar lavage (BAL) in children with refractory $M$. pneumoniae pneumonia with radiologic evidence of large pulmonary lesions reduced fever, corrected laboratory indices, and provided symptomatic relief. Early BAL in refractory $M$. pneumoniae pneumonia should be considered.

cluded patients with other respiratory tract infections and tuberculosis by the following tests: purified protein derivative, blood cultures, nasopharyngeal aspirate/swab cultures, nasopharyngeal aspirate/swab for virus antigens detection (respiratory syncytial viruses, influenza viruses, adenovirus, and parainfluenza virus), and serology for Chlamydia pneumoniae and Legionella pneumophila. Patients who received corticosteroids before admission or had underlying diseases such as chronic cardiac and pulmonary disease and immunodeficiency were also excluded.

\section{Study Design}

This was an observational prospective study. All of the enrolled subjects had indications for FOB and BAL because of radiologically proven large pulmonary lesions and unresponsiveness to routine antibiotic therapy. Once parental consent was obtained and preoperative preparations were completed, the BAL was performed. According to the time point of BAL, they were divided into 2 groups. The early BAL group subjects received BAL within $3 \mathrm{~d}$ after admission (including the third day of hospitalization), whereas the late BAL group had this procedure $>3 \mathrm{~d}$ after admission. Both groups received similar supportive and symptomatic treatments, including sputum aspiration, nebulization, and fluid therapy. Subjects were discontinued from the study if they met exclusion criteria during the hospitalization, if their parents were unwilling for them to remain as study subjects, or if they were discharged before the end of study.

The study was approved by the ethics committee of the Children's Hospital, Zhejiang University School of Medicine. All parents or legal guardians provided written in- 
formed consent before conducting the study and any studyrelated procedures.

\section{BAL Under Flexible Bronchoscopy}

According to the age and body weight of the subjects, 3 kinds of FOB were used as follows: Olympus (Japan) BFXP40 (2.8 mm external diameter and $1.2 \mathrm{~mm}$ working channel), BF-3C30 (3.6 mm, $1.2 \mathrm{~mm}$ ), and BF-P40 (4.9 mm, $2.2 \mathrm{~mm}$ ). After fasting for $>6 \mathrm{~h}$, the subjects were sedated with intravenous midazolam $(0.1-0.15 \mathrm{mg} / \mathrm{kg})$, and topical anesthesia with $1 \%$ lidocaine to the nose, vocal cords, and trachea was added. The flexible bronchoscope was wedged in the orifice of lobar bronchi where the large pulmonary lesion was localized on chest radiograph. BAL with normal saline (weight $<20 \mathrm{~kg}: 1 \mathrm{~mL} / \mathrm{kg} / \mathrm{time}, 3$ times; weight $>20 \mathrm{~kg}: 20 \mathrm{~mL} / \mathrm{time}, 3$ times) was conducted with -25 to approximately $-100 \mathrm{~mm} \mathrm{Hg}$ suction. We also collected BAL fluid from subjects for microbiological determinations, as we previously reported. ${ }^{24,25}$ During the procedure, we monitored the heart rate, breathing frequency, and pulse oxygen saturation $\left(\mathrm{S}_{\mathrm{pO}_{2}}\right)$. Once hypoxia (cyanosis, low $\mathrm{S}_{\mathrm{pO}_{2}}$, and/or high heart rate) occurred, oxygen of appropriate concentration was given at once, and the procedure was stopped temporarily when necessary.

\section{Data Collection}

The following data before and during hospitalization were recorded: demographics; fever duration before admission; duration of macrolide therapy before admission; the incidence of severe $M$. pneumoniae pneumonia and extrapulmonary complications; the presence of lobar atelectasis and pleural effusion; and the results of blood tests including white blood cell (WBC) count, the differential of neutrophils as well as lymphocytes, C-reactive protein, procalcitonin, and lactate dehydrogenase. All subjects were followed until discharge from hospital. Body temperature and respiratory tract signs and symptoms of subjects were examined at study entry and every $8 \mathrm{~h}$ thereafter. A febrile day was defined as a day during which the body temperature exceeded $38.0^{\circ} \mathrm{C}$ at least once. ${ }^{26}$ Febrile days after admission and hospitalized days were also assessed. The WBC count and C-reactive protein were detected at study entry and every 2-3 d thereafter. All subjects underwent chest $\mathrm{X}$-rays before admission and again approximately $7 \mathrm{~d}$ after admission. The severity of pneumonia was evaluated according to the diagnostic standard of pneumonia advocated by British Thoracic Society. ${ }^{27}$ During the hospitalization, we also evaluated the extrapulmonary complications (eg, liver function abnormalities, myocarditis, encephalitis, and rash) of subjects. ${ }^{28}$ The safety of subjects

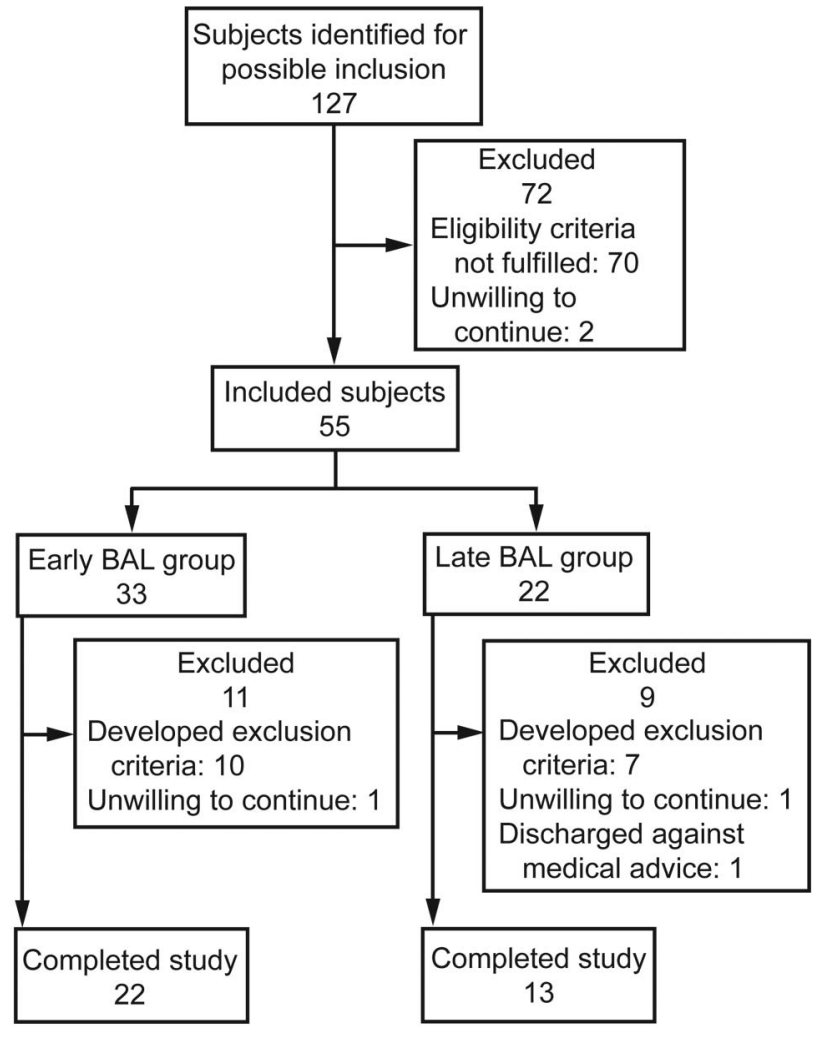

Fig. 1. Study flow chart. BAL $=$ bronchoalveolar lavage.

was also evaluated by recording adverse events during hospitalization.

\section{Statistical Analysis}

Statistical analysis was performed using SPSS 15.0 software (SPSS, Chicago, Illinois). Normally distributed data were expressed as mean $\pm \mathrm{SD}$. An independent-sample $t$ test was used to compare these data. Data with a skewed distribution were expressed as median values (range from minimum to maximum). The comparisons were made by Mann-Whitney U test. Chi-square tests were used to compare categorical data. Statistical significance was defined as $P<.05$.

\section{Results}

\section{General Information and Clinical Data}

One hundred and twenty-seven subjects were identified as suffering from refractory $M$. pneumoniae pneumonia and therefore potentially eligible for the study. We excluded 70 of those subjects because they did not fulfill the inclusion criteria, and 2 subjects were not willing to continue. Twenty subjects did not complete the study because they developed exclusion criteria, were unwilling to con- 
Table 1. Clinical Information of Refractory Mycoplasma pneumoniae Pneumonia Subjects Before BAL Therapy

\begin{tabular}{|c|c|c|c|c|}
\hline Clinical information & $\begin{array}{l}\text { Total Subjects } \\
\quad(n=35)\end{array}$ & $\begin{array}{l}\text { Early BAL Group } \\
\quad(n=22)\end{array}$ & $\begin{array}{l}\text { Late BAL Group } \\
\quad(n=13)\end{array}$ & $P$ \\
\hline Age $(y)$ & $7.3 \pm 2.8$ & $7.6 \pm 2.7$ & $6.8 \pm 3.0$ & .41 \\
\hline Male/female & $20 / 15$ & $12 / 10$ & $8 / 5$ & .69 \\
\hline Fever duration prior to admission (d)* & $8(7-21)$ & $8(7-21)$ & $8(7-13)$ & .78 \\
\hline Duration of macrolide therapy before admission (d)* & $7(7-13)$ & $7(7-13)$ & $7(7-9)$ & .43 \\
\hline $\mathrm{WBC}\left(\times 10^{9} / \mathrm{L}\right)^{*}$ & $6.91(1.98-21.30)$ & $6.48(3.59-21.30)$ & $7.77(1.98-14.68)$ & .89 \\
\hline $\mathrm{N}(\%)^{*}$ & $73.0(24.7-86.5)$ & $70.9(28.9-82.2)$ & $77.5(24.7-86.5)$ & .35 \\
\hline $\mathrm{L}(\%)^{*}$ & $18.9(8.1-72.3)$ & $20.0(10.5-63.8)$ & $15.3(8.1-72.3)$ & .15 \\
\hline C-reactive protein $(\mathrm{mg} / \mathrm{dL})^{*}$ & $37(2-160)$ & $45(4-160)$ & $28(2-160)$ & .05 \\
\hline Procalcitonin $(\mathrm{ng} / \mathrm{mL})^{*}$ & $0.20(0.06-5.46)$ & $0.17(0.06-3.21)$ & $0.45(0.09-5.46)$ & .65 \\
\hline LDH (IU/L)* & $494(110-2159)$ & $461(165-893)$ & $503(110-2159)$ & .28 \\
\hline Subjects with lobar atelectasis on chest $\mathrm{x}$-ray $(\%) \dagger$ & $46(16 / 35)$ & $41(9 / 22)$ & $54(7 / 13)$ & .46 \\
\hline Subjects with pleural effusion on chest $\mathrm{x}$-ray $(\%) \dagger$ & $51(18 / 35)$ & $50(11 / 22)$ & $54(7 / 13)$ & .83 \\
\hline Subjects with severe $M$. pneumoniae pneumonia $(\%) \dagger$ & $29(10 / 35)$ & $32(7 / 22)$ & $23(3 / 13)$ & .58 \\
\hline Subjects presenting with extrapulmonary complications $(\%) \dagger$ & $54(19 / 35)$ & $50(11 / 22)$ & $62(8 / 13)$ & .51 \\
\hline Digestive system (liver function abnormalities) $\dagger$ & $53(10 / 19)$ & $55(6 / 11)$ & $50(4 / 8)$ & \\
\hline Cardiovascular system (myocarditis) $\dagger$ & $16(3 / 19)$ & $18(2 / 11)$ & $13(1 / 8)$ & \\
\hline $\operatorname{Rash} \dagger$ & $26(5 / 19)$ & $27(3 / 11)$ & $25(2 / 8)$ & \\
\hline Nervous system (encephalitis) $\dagger$ & $5(1 / 19)$ & $0(0 / 11)$ & $13(1 / 8)$ & \\
\hline $\begin{array}{l}\text { LDH normal range: } 120-300 \mathrm{IU} / \mathrm{L} . P, \text { group } 1 \text { vs group } 2 . \\
\text { * Data are shown as median (range). } \\
\dagger \text { Data are shown as percentage }(n) . \\
\text { BAL = bronchoalveolar lavage } \\
\text { WBC = white blood cells } \\
\mathrm{N}=\text { neutrophils } \\
\mathrm{L}=\text { lymphocytes } \\
\mathrm{LDH}=\text { lactate dehydrogenase }\end{array}$ & & & & \\
\hline
\end{tabular}

tinue, or left the hospital against medical advice. The remaining 35 subjects completed the study (Fig. 1).

Clinical information of enrolled subjects before admission is shown in Table 1 . The mean age of the 35 subjects was $7.3 \pm 2.8 \mathrm{y}$ (range, $1-13 \mathrm{y}$ ). Twenty subjects were male. The median prior-to-admission duration of fever was 8 (7-21) d. The median WBC count on admission was $6.91(1.98-21.30) \times 10^{9} / \mathrm{L}$ with $73.0 \%(24.7-86.5 \%)$ neutrophils and $18.9 \%(8.1-72.3 \%)$ lymphocytes. The median value of C-reactive protein was 37 (2-160) $\mathrm{mg} / \mathrm{dL}$, which was severalfold higher than normal reference. The median levels of procalcitonin and lactate dehydrogenase on admission were $0.20(0.06-5.46) \mathrm{ng} / \mathrm{mL}$ and $494(110-2,159)$ IU/L, respectively. Of the 35 subjects, extrapulmonary complications were found in 19 cases (54\%), including liver function abnormalities in 10, myocarditis in 3 , rash in 5 , and encephalitis in 1 . We also evaluated the severity of $M$. pneumoniae pneumonia and found the incidence of severe M. pneumoniae pneumonia was $29 \%$ in all subjects. However, none of these subjects was transferred to the ICU or given mechanical ventilation during hospitalization. The median hospitalization duration was 7 (5-14) d. In addition to clinical symptoms, radiological findings were also evaluated. We found that $46 \%$ of the subjects showed lobar atelectasis and $51 \%$ of the subjects showed pleural effusion on their chest x-rays.

Among the 35 subjects, 34 yielded positive results for both $M$. pneumoniae immunoglobulin $\mathrm{M}$ in serum and polymerase-chain-reaction test in nasopharyngeal secretions and/or BAL. One subject had positive $M$. pneumoniae immunoglobulin M only. M. pneumoniae polymerase chain reaction was positive in 31 subjects (89\%) using BAL specimens, and in 22 subjects $(63 \%)$ using nasopharyngeal secretions $(P=.01)$.

Baseline characteristics of the early BAL group and the late BAL group were compared in Table 1. Twenty-two subjects were enrolled in the early BAL group (12 males, 10 females), with a mean age of $7.6 \pm 2.7 \mathrm{y}$ (range, 1-13 y). Thirteen subjects were enrolled in the late BAL group ( 8 males, 5 females), with a mean age of $6.8 \pm 3.0 \mathrm{y}$ (range, 2-11 y). The median time lag from admission to BAL procedure was $2.5(1-3) \mathrm{d}$ in the early BAL group and 5 (4-12) $d$ in the late BAL group. Between these 2 groups, there were no significant differences in these baseline data, including fever duration before admission, duration of macrolide treatment before admission, laboratory 
Table 2. Clinical Information of Refractory Mycoplasma pneumoniae Pneumonia Subjects After BAL Therapy

\begin{tabular}{|c|c|c|c|c|}
\hline Information & $\begin{array}{l}\text { Total Subjects } \\
\quad(N=35)\end{array}$ & $\begin{array}{c}\text { Early BAL Group } \\
\quad(n=22)\end{array}$ & $\begin{array}{l}\text { Late BAL Group } \\
\qquad(n=13)\end{array}$ & $P$ \\
\hline Fever duration after admission $(\mathrm{d}) *$ & $4(2-10)$ & $4(2-7)$ & $5(2-10)$ & .03 \\
\hline Median hospitalization duration $(\mathrm{d})^{*}$ & $7(5-14)$ & $7(5-10)$ & $10(5-14)$ & .02 \\
\hline Median time for WBC count recovered (d)* & $6(1-13)$ & $6(1-7)$ & $8(1-13)$ & .003 \\
\hline Median time for C-reactive protein recovered (d)* & $7(1-13)$ & $6(1-7)$ & $8(1-13)$ & .001 \\
\hline Atelectasis resolution rate $(\%) \dagger$ & $44(7 / 16)$ & $67(6 / 9)$ & $14(1 / 7)$ & .04 \\
\hline Pleural effusion disappearance rate $(\%) \dagger$ & $39(7 / 18)$ & $55(6 / 11)$ & $14(1 / 7)$ & .09 \\
\hline Subjects with mucus plugs under bronchoscope $(\%) \dagger$ & $20(7 / 35)$ & $14(3 / 22)$ & $31(4 / 13)$ & .22 \\
\hline $\begin{array}{l}\text { Subjects with positive result for } M . \text { pneumoniae } \\
\text { polymerase chain reaction of BAL }(\%) \dagger\end{array}$ & $89(31 / 35)$ & $96(21 / 22)$ & $77(10 / 13)$ & .10 \\
\hline \multicolumn{5}{|c|}{$\begin{array}{l}P \text {, group } 1 \text { vs group } 2 \text {. We evaluated the atelectasis resolution rate and pleural effusion disappearance rate } \sim 7 \mathrm{~d} \text { after admission (if the hospitalization duration was }<7 \mathrm{~d} \text {, we evaluated on the day of } \\
\text { discharge). } \\
* \text { Data are shown as median (range). } \\
\uparrow \text { Data are shown as percentage }(n) . \\
\text { BAL }=\text { bronchoalveolar lavage } \\
\text { WBC }=\text { white blood cells }\end{array}$} \\
\hline
\end{tabular}

abnormalities, severity of radiographic findings, incidence of extrapulmonary complications, and incidence of severe M. pneumoniae pneumonia, as shown in Table 1 .

\section{Clinical, Laboratory, and Radiological Effectiveness}

Among 35 subjects who received bronchoscopy, bronchial membrane inflammation was noted in all subjects, whereas mucus plug was noted only in 7 subjects. As shown in Table 2, we found that there were no significant differences between the 2 groups in the incidence of mucus plug ( $14 \%$ vs $31 \%, P=.22$ ). We also compared the positive rate for $M$. pneumoniae of BAL in all subjects, and found that the positive rate for $M$. pneumoniae was $96 \%$ in the early BAL group and $77 \%$ in the late BAL group, with no significant difference $(P=.10)$.

After BAL therapy, symptom relief was obtained in all subjects. Peak body temperature decreased significantly within $24 \mathrm{~h}$ in all subjects, and body temperature returned to normal in 28 (out of 35) subjects within $2 \mathrm{~d}$. The median duration of fever after BAL therapy was $1(0-6) \mathrm{d}$. Moreover, rapid resolution of atelectasis in pulmonary lobes was documented on radiography (Fig. 2).

Comparing data between the 2 groups, we found that the median duration of fever after admission was shorter in the early BAL group $(4[2-7] \mathrm{d})$ than that in the late BAL group $(5[2-10] \mathrm{d})(P=.03)$. The early BAL group's median hospitalization duration was $7(5-10) \mathrm{d}$ versus the late BAL group's $10(5-14) \mathrm{d}(P=.02)$. As shown in Table 2, we also found that WBC count and C-reactive protein recovered more quickly in the early $\mathrm{BAL}$ group than those in the late BAL group $(P=.003, P=.001$, respectively). In addition to clinical symptoms, we found improved radiological manifestations in the early BAL

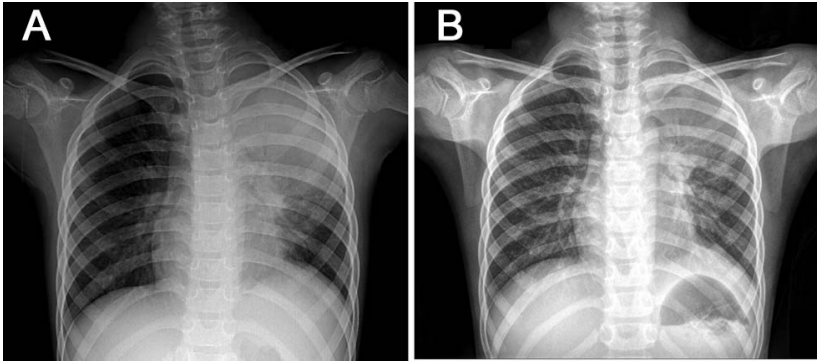

Fig. 2. Chest radiographies of case 1 (8-y-old female). A: on admission; B: $7 \mathrm{~d}$ after bronchoalveolar lavage therapy.

group compared to the late BAL group. Approximately $7 \mathrm{~d}$ after admission, $67 \%$ of subjects in the early BAL group showed atelectasis resolution versus $14 \%$ in the late BAL group $(P=.04)$. A higher rate of pleural effusion disappearance was also seen in the early BAL group when compared with the late BAL group (55\% vs $14 \%$ ), but the difference did not reach statistical significance $(P=.09)$.

\section{Adverse Events}

There were no obvious adverse events in any subjects during the BAL procedure. After performing BAL, no deterioration in clinical manifestations, radiographic findings, or laboratory results was observed in any subjects from either group. All subjects were discharged without morbidity.

\section{Discussion}

Although M. pneumoniae infection was traditionally thought to be a self-limited process, increasing numbers of severe and even fatal cases of $M$. pneumoniae infections 
have been reported. ${ }^{3-9}$ Reports of cases of refractory M. pneumoniae pneumonia, which displays clinical and radiological progression after macrolide therapy for $7 \mathrm{~d}$ or longer, are increasing as well. ${ }^{11,14,15}$

The reasons why refractory $M$. pneumoniae pneumonia occurs remain unclear, but it is widely accepted that immunological response mediated by $\mathrm{T}$ cells seems to play an important role in the progress of refractory M. pneumoniae pneumonia. Animal studies showed that the peribronchiolar and perivascular regions were initially infiltrated by a large number of lymphocytes, mainly $\mathrm{CD}_{4} \mathrm{~T}$ cells when the animals were infected with M. pneumoniae. ${ }^{29}$ It was also demonstrated that the levels of serum interleukin-18, which promoted Th1 responses, were higher in severe M. pneumoniae infection than those in mild cases. ${ }^{30}$ Thus, stronger immunological reactions may underlie more severe pathological infiltration in the lung. In our study, the elevated C-reactive protein may have indicated severe systemic inflammatory response to $M$. pneumoniae infection.

Given that the M. pneumoniae infection was an immunity-mediated disease, clinicians could adopt immunosuppressive therapy to treat it. There have been some reports demonstrating that corticosteroids were used for refractory M. pneumoniae pneumonia in children with satisfactory results. Tamura et al ${ }^{11}$ reported that it was apparently an efficacious and well-tolerated treatment to administer methylprednisolone intravenously with a dosage of $30 \mathrm{mg} / \mathrm{kg} / \mathrm{d}$ for 3 consecutive days for refractory M. pneumoniae. Luo et al ${ }^{14}$ demonstrated that oral prednisolone of $2 \mathrm{mg} / \mathrm{kg} / \mathrm{d}$ combined with intravenously azithromycin was helpful for children with refractory $M$. pneumoniae pneumonia.

However, tuberculosis is still a heavy burden in China, and it should be taken into account when differential diagnosis is made for refractory pneumonia. Some patients may be simultaneously infected with $M$. pneumoniae and tuberculosis, and sometimes transient tuberculin anergy may occur in M. pneumoniae infection. Therefore, clinicians should rule out tuberculosis as a potential cause of pulmonary infiltrates, especially in refractory pneumonia, and prescribe corticosteroid administration with caution. Because it will take several days to exclude tuberculosis, delayed use of corticosteroids might result in longer fever duration and more severe pulmonary inflammation. Therefore, we should find a new way to help control local inflammation in refractory $M$. pneumoniae pneumonia.

Currently, FOB with BAL is an important and indispensable tool to assess the structure and function of lower airways and to diagnose pulmonary infiltrative lesions in pediatric patients. Lacroix et $\mathrm{al}^{31}$ have demonstrated that BAL was safe and more efficient than blood culture to detect pathogens in patients presenting with pneumonia. In our results, we found that BAL was a more effective method to identify pathogens than nasopharyngeal secretion de- tection ( $89 \%$ vs $63 \%, P=.01)$. Furthermore, endobronchial tuberculosis could be excluded earlier. Additionally, FOB could also perform therapeutic interventions. Several studies have shown that FOB with BAL was the preferred treatment for lobar atelectasis. ${ }^{32,33}$ Abu-Hasan et al ${ }^{32}$ suggested that BAL could be safe and effective in treating acute lung collapse and atelectasis that was refractory to conventional therapy. To our knowledge, this is the first study to focus on the effect of BAL in patients with refractory M. pneumoniae pneumonia in a prospective fashion. In our study, we evaluated the effect of BAL on refractory M. pneumoniae pneumonia with large infiltrative lesions in 35 subjects, and analyzed the impact of application time on the curative effect. Interestingly, we found that after BAL therapy peak body temperature in all subjects decreased significantly within $24 \mathrm{~h}$, and radiological as well as laboratory abnormalities recovered rapidly. Furthermore, our study showed that early application of BAL could ameliorate clinical symptoms faster, and improve radiographic and laboratory findings earlier than late application. After starting the therapy, body temperature for subjects in the early BAL group returned to normal within $4(2-7) \mathrm{d}$ versus $5(2-10) \mathrm{d}$ in the late BAL group $(P<.05)$. Hospitalization duration was shortened and more subjects obtained atelectasis resolution in the early BAL group than in the late BAL group $(P<.05)$. Peripheral WBC count and $\mathrm{C}$-reactive protein recovered more quickly in the early BAL group $(P<.01)$. However, our study involved only a small number of subjects, and a larger prospective study is needed to confirm the role of BAL therapy in refractory M. pneumoniae pneumonia.

\section{Conclusions}

We performed an observational prospective study evaluating the effect of BAL in children with refractory M. pneumoniae pneumonia and radiologically proven large pulmonary lesions. The BAL intervention demonstrated a positive outcome with rapid improvement in clinical, radiographic, and laboratory results. Early application of BAL may render greater benefit in curing children with refractory $M$. pneumoniae pneumonia and large pulmonary lesions.

\section{ACKNOWLEDGMENTS}

We are grateful to our colleagues in the Department of Pulmonology for attending the subjects who participated in this study.

\section{REFERENCES}

1. Michelow IC, Olsen K, Lozano J, Rollins NK, Duffy LB, Ziegler T, et al. Epidemiology and clinical characteristics of community-acquired pneumonia in hospitalized children. Pediatrics 2004;113(4): 701-707. 


\section{Bronchoalveolar Lavage in Pneumonia}

2. Defilippi A, Silvestri M, Tacchella A, Giacchino R, Melioli G, Di Marco E, et al. Epidemiology and clinical features of Mycoplasma pneumoniae infection in children. Resp Med 2008;102(12):17621768.

3. Christie LJ, Honarmand S, Talkington DF, Gavali SS, Preas C, Pan $\mathrm{CY}$, et al. Pediatric encephalitis: what is the role of Mycoplasma pneumoniae? Pediatrics 2007;120(2):305-313.

4. Azumagawa K, Kambara Y, Murata T, Tamai H. Four cases of arthritis associated with Mycoplasma pneumoniae infection. Pediatr Int 2008;50(4):511-513.

5. Hawkins S, Rausch CM, McCanta AC. Constrictive pericarditis secondary to infection with Mycoplasma pneumoniae. Curr Opin Pediatr 2011;23(1):126-129.

6. Khan FY, Ayassin M. Mycoplasma pneumoniae associated with severe autoimmune hemolytic anemia: case report and literature review. Braz J Infect Dis 2009;13(1):77-79.

7. Wang RS, Wang SY, Hsieh KS, Chiou YH, Huang IF, Cheng MF, Chiou CC. Necrotizing pneumonitis caused by Mycoplasma pneumoniae in pediatric patients. Pediatr Infect Dis J 2004;23(6):564567.

8. Radisic M, Torn A, Gutierrez P, Defranchi HA, Pardo P. Severe acute lung injury caused by Mycoplasma pneumoniae: potential role for steroid pulses in treatment. Clin Infect Dis 2000;31(6):15071511.

9. Takiguchi Y, Shikama N, Aotsuka N, Koseki H, Terano T, Hirai A. Fulminant Mycoplasma pneumoniae pneumonia. Intern Med 2001; 40(4):345-348.

10. The Subspecialty Group of Respiratory Disease, The Society of Pediatrics, Chinese Medical Association, The Editorial Board, Chinese Journal of Pediatrics. Guidelines for management of community acquired pneumonia in children (the revised edition of 2013) (I). Chin J Pediatr 2013;51(10):745-749.

11. Tamura A, Matsubara K, Tanaka T, Nigami H, Yura K, Fukaya T. Methylprednisolone pulse therapy for refractory Mycoplasma pneumoniae pneumonia in children. J Infect 2008;57(3):223-228.

12. Fernald GW. Immunologic mechanisms suggested in the association of $M$. pneumoniae infection and extrapulmonary disease: a review. Yale J Biol Med 1983;56(5-6):475-479.

13. Waites KB, Talkington DF. Mycoplasma pneumoniae and its role as a human pathogen. Clin Microbiol Rev 2004;17(4):697-728.

14. Luo Z, Luo J, Liu E, Xu X, Liu Y, Zeng F, et al. Effects of prednisolone on refractory Mycoplasma pneumoniae pneumonia in children. Pediatr Pulmonol 2014;49(4):377-380.

15. Lee KY, Lee HS, Hong JH, Lee MH, Lee JS, Burgner D, Lee BC. Role of prednisolone treatment in severe Mycoplasma pneumoniae pneumonia in children. Pediatr Pulmonol 2006;41(3):263-268.

16. Wood RE, Fink RJ. Applications of flexible fiberoptic bronchoscopes in infants and children. Chest 1978;73(5 Suppl):737-740.

17. Nussbaum E. Pediatric fiberoptic bronchoscopy: clinical experience with 2836 bronchoscopies. Pediatr Crit Care Med 2002;3(2):171176.

18. Efrati O, Sadeh-Gornik U, Modan-Moses D, Barak A, Szeinberg A, Vardi A, et al. Flexible bronchoscopy and bronchoalveolar lavage in pediatric patients with lung disease. Pediatr Crit Care Med 2009; 10(1):80-84
19. Shah MB, Bent JP, Vicencio AG, Veler H, Arens R, Parikh SR. Flexible bronchoscopy and interdisciplinary collaboration in pediatric large airway disease. Int J Pediatr Otorhinolaryngol 2008;72(12): 1771-1776

20. Naidich DP, Harkin TJ. Airways and lung: correlation of CT with fiberoptic bronchoscopy. Radiology 1995;197(1):1-12.

21. Kapur N, Slater A, McEniery J, Greer ML, Masters IB, Chang AB Therapeutic bronchoscopy in a child with sand aspiration and respiratory failure from near drowning-case report and literature review. Pediatr Pulmonol 2009;44(10):1043-1047.

22. Nelson S, Belknap SM, Carlson RW, Dale D, DeBoisblanc B, Farkas $\mathrm{S}$, et al. A randomized controlled trial of filgrastim as an adjunct to antibiotics for treatment of hospitalized patients with communityacquired pneumonia. CAP Study Group. J Infect Dis 1998;178(4): 1075-1080

23. Uehara S, Sunakawa K, Eguchi H, Ouchi K, Okada K, Kurosaki T, et al. Japanese guidelines for the management of respiratory infectious diseases in children 2007 with focus on pneumonia. Pediatr Int 2011;53(2):264-276

24. Tang LF, Xu YC, Wang YS, Wang CF, Zhu GH, Bao XE, et al. Airway foreign body removal by flexible bronchoscopy: experience with 1027 children from 2000-2008. World J Pediatr 2009;5(3):191195.

25. Tang LF, Chen ZM. Fiberoptic bronchoscopy in neonatal and pediatric intensive care units: a 5-year experience. Med Princ Pract 2009; 18(4):305-309.

26. Suzuki S, Yamazaki T, Narita M, Okazaki N, Suzuki I, Andoh T, et al. Clinical evaluation of macrolide-resistant Mycoplasma pneumoniae. Antimicrob Agents Chemother 2006;50(2):709-712.

27. British Thoracic Society of Standards of Care Committee. British Thoracic Society guidelines for the management of community acquired pneumonia in childhood. Thorax 2002;57(suppl):S1-S34.

28. Zhou Y, Zhang Y, Sheng Y, Zhang L, Shen Z, Chen Z. More complications occur in macrolide-resistant than in macrolide-sensitive Mycoplasma pneumoniae pneumonia. Antimicrob Agents Chemother 2014;58(2):1034-1038

29. Opitz O, Pietsch K, Ehlers S, Jacobs E. Cytokine gene expression in immune mice reinfected with Mycoplasma pneumoniae: the role of $\mathrm{T}$ cell subsets in aggravating the inflammatory response. Immunobiology 1996-1997; 196(5):575-587

30. Tanaka H, Narita M, Teramoto S, Saikai T, Oashi K, Igarashi T, Abe S. Role of interleukin-18 and T-helper type 1 cytokines in the development of Mycoplasma pneumoniae pneumonia in adults. Chest 2002;121(5):1493-1497.

31. Lacroix G, Prunet B, Bordes J, Cabon-Asencio N, Asencio Y, Gaillard T, et al. Evaluation of early mini-bronchoalveolar lavage in the diagnosis of health care-associated pneumonia: a prospective study. Crit Care 2013;17(1):R24

32. Abu-Hasan MN, Chesrown SE, Jantz MA. Successful use of bronchoscopic lung insufflation to treat left lung atelectasis. Pediatr Pulmonol 2013;48(3):306-309.

33. Kreider ME, Lipson DA. Bronchoscopy for atelectasis in the ICU: a case report and review of the literature. Chest 2003;124(1):344350. 